\title{
Adjunctive Therapies for Treatment of Severe Respiratory Failure in Neonates
}

\section{Ergänzende Therapien bei Neugeborenen mit schwerem respiratorischen Versagen}

Ludwig Gortner

Bibliography

DOI http://dx.doi.org/

$10.1055 / \mathrm{s}-0035-1547238$

Klin Padiatr 2015; 227: 51-53

(C) Georg Thieme Verlag KG

Stuttgart · New York

ISSN 0300-8630

Korrespondenzadresse

Prof. Dr. Ludwig Gortner

Clinics for Paediatrics and

Adolescent Medicine

University Hospital of the

Saarland

Building 9

66421 Homburg

Germany

ludwig.gortner@uks.eu
Severe neonatal pulmonary failure in late preterm or term neonates still represents a major challenge in neonatology. In contrary with a considerable number of controlled clinical trials facing classical neonatal respiratory distress syndrome (RDS), e.g. primary surfactant deficiency syndrome in an immature lung, only a limited number of studies have been published to address this problem in near term or term neonates. Since the beginning $90 \mathrm{~s}$, extracorporeal membrane oxygenation (ECMO) had been getting standard in neonatal intensive care. This trend had been intensified since the publication of a clinical controlled trial and follow-up data from the UK, testing ECMO in term neonates with severe respiratory failure [3].

In parallel with this trend, adjunctive therapies in severe neonatal respiratory failure have been developed facing surfactant administration, e.g. for meconium aspiration syndrome [11], administration of nitric oxide in persistent pulmonary hypertension [4] and high-frequency oscillation ventilation in hypoxic respiratory failure [8]. The data of these studies were convincing with respect to improving gas exchange acutely after introduction of either therapy, however, upon analyses of survival without oxygen requirements or other major clinical outcome variables, only few studies did show efficacy in term neonates suffering from either pathophysiology of respiratory failure.

\section{Surfactant in meconium aspiration syndrome \\ $\nabla$}

Meconium aspiration syndrome is caused by aspiration of meconium before and during birth a leads to obstruction of small airways, inflammation and inactivation of surfactant. The frequency of this severe perinatal complication leading potentially to a terminal respiratory failure with the need for ECMO has been decreasing over the last decades [15]. During the $90 \mathrm{~s}$, several studies have been published, investigation the effects of surfactant administration either for lung lavage or after lavage just for replacement.The usage of surfactant in neonatal meconium aspiration syndrome in term or preterm infants had been analyzed in Cochrane analysis lately, with only 2 clinical controlled trials: The results indicate a relative risk for the need of ECMO of 0.64, confidence interval $0.46-0.91$. However, there were no further significant reductions in other neonatal outcome variables including mortality, duration of mechanical ventilation, oxygen supplement as well as the rate of pneumothorax, pul- monary interstitial emphysema and other forms of neonatal air leaks [10]. Pulmonary air leaks have been reported to overall complicate mechanical ventilation in late preterm and term neonates [19]. As pulmonary surfactant replacement therapy is standard in the treatment of classical neonatal respiratory distress syndrome [23], it may be considered in specific pre-ECMO conditions.

\section{Nitric oxide in persistent pulmonary hypertension of the neonate \\ $\nabla$}

The usage of inhaled nitric oxide in term and near-term neonates had been addressed in a meta-analysis published by 2006 with 14 trials, which were eligible [12]. Oxygenation was improved after introduction of inhaled nitric oxide by about one half, and correspondingly, the oxygenation index decreased at least in infants with neonatal RDS suffering from coexisting persistent pulmonary hypertension of the neonate (PPHN) significantly. However, in the subgroup of babies with congenital diaphragmatic hernia, no significant improvement was observed. This subgroup of neonates presenting often with severe neonatal respiratory failure has been reported to differ considerably from other subgroups with respect to different response to interventions for treating severe neonatal respiratory failure [22]. As a conclusion from the trials on nitric oxide, it does seem reasonable to analyze term neonates or late preterms not only on the basis of need of adjunctive therapies, but also to select carefully underlying disorders leading to severe neonatal respiratory failure. Different pathophysiologies of severe respiratory failure in this distinct age group yield various therapeutic responses depending on the underlying disorder.

In the group of preterm neonates, a meta-analysis was published in 2011 reporting on about 2300 preterms in 11 trials. There was no effect of the routine use of inhaled nitric oxide in these cohorts of preterm neonates with respect to typical neonatal outcome variables: mortality, bronchopulmonary dysplasia (BPD) or severe neurologic disorders including intracranial hemorrhage and periventricular leukomalacia [1].

It thus can be concluded for the use of inhaled nitric oxide that in term neonates with severe respiratory failure excluding congenital diaphragmatic hernia the use of nitric oxide as an adjunctive therapy can be recommended. However, this does not hold true in preterm neonates, mostly suffering from classical neonatal RDS. 


\section{High frequency oscillation ventilation in neonatal respiratory failure \\ $\nabla$}

As the third major adjunctive therapy, high-frequency ventilation in preterm neonates with RDS has been studied in a number of clinical controlled trials, which were summarized in a metaanalysis published in 2009 enrolling about 3700 neonates [9]. This very carefully conducted meta-analysis did not show any evidence for the superiority of high-frequency ventilation on neonatal mortality, e.g. 28 days mortality in this study; the authors further performed the analyses of subgroups showing a significant reduction in the rate of BPD when high-frequency oscillation was used with surfactant or when piston oscillators were applied or when lung protective strategies for conventional ventilation was not used. Thus, the authors conclude that there is no convincing evidence to indicate priority of high-frequency oscillation in neonatal RDS in preterm neonates.

Only scarce studies are available for the effect of high-frequency ventilation in term neonates: the actual meta-analysis of highfrequency oscillation in this group only enrolls 2 trials [14] where HFOV was either compared with conventional ventilation with or without a rescue cross over design. In this study, neonates with severe respiratory failure were started either on conventional ventilation or rescue high-frequency oscillation. There was no evidence that either as rescue therapy or as a primary mode of ventilation, high-frequency oscillation improves major neonatal outcome variables, e.g. mortality or survival without oxygen requirements at day 28 , the number of air leaks, time on mechanical ventilation and long term oxygen dependency. The same holds true for a study reviewing data in post-neonatal respiratory failure during childhood. Thus no clear evidence could be found for the usage of high-frequency oscillation reducing mortality or duration of ventilation or chronic lung disease in term neonates or older children with severe respiratory failure. The evidence of the effects of HFOV is highest in preterm neonates, whereas in term neonates there is a gross lack of data indicated by a low number of individuals enrolled in the aforementioned meta-analysis [14].

\section{ECMO in the treatment of severe respiratory failure in neonates \\ $\nabla$}

After establishing ECMO as a lifesaving therapy in neonates in the $90 \mathrm{~s}$ of the past century [2] there had been a spreading of this technique, not only being administered to neonates with severe respiratory failure, but also in the postsurgical treatment of neonates with congenital heart disease. This group of neonates actually may represent the largest group receiving ECMO during the neonatal period, but also for neonates or young infants for further cardiac failure due to myocarditis and other infectious disorders [21]. Thus, establishing ECMO did offer a substantial improvement in neonates with severe cardiac as well as pulmonary failure regardless of the underlying disorders. Data from Germany [21] as well as from the United States [13] indicate that ECMO overall is a very successful method. However, the data from US indicate that prolonged ECMO therapy, e.g. administration $>28$ days in neonates with pulmonary or cardiac end stage failure is not overall successfully [14]. Thus, there seems to be a broad consensus that neonates with otherwise not treatable respiratory failure including those with septic shock [5] should be considered as candidates for ECMO. Thus, given a robust set of data indicating the usefulness for ECMO in most clinical settings applied to neonates with intractable cardiopulmonary failure, further steps include the evaluation of adjunctive therapies.

Given these facts, the authors of a recently published paper [18] use the instrument of "Erhebungseinheit für seltene pädiatrische Erkrankungen” (ESPED), where somewhat less than 400 near term or term neonates over a 2-year-period are enrolled, who were given ECMO or considered to be in a pre-ECMO condition. Underlying disorders were reported to be respiratory distress syndrome in somewhat more than one third of the patients, followed by pneumonia and sepsis (16\%), further meconium aspiration syndrome and congenital diaphragmatic hernia. The latter group seems to be overrepresented due to the fact that most reports were given from the Mannheim ECMO center. The authors clearly show that most patients (77\%) were given surfactant with a success rate of somewhat more than $70 \%$. Somewhat more than $40 \%$ of all patients were treated with inhaled nitric oxide, which led to a success rate of about $50 \%$ and about one third was treated with high-frequency ventilation, where a response was noted in about $60 \%$.

Thus, these data should be interpreted on the background of the meta-analyses, which had been given before. Given the fact, that only limited data are available on the basis of rigorously controlled clinical trials, the results should be interpreted at least with some caution. A major problem with interpretation of the data is represented by the fact that a group of patients enrolled in the ESPED trial on adjunctive therapies for treatment for severe respiratory failure is influenced of cause of respiratory failure present in various subgroups.

Thus, surfactant administration in a baby judged to suffer from neonatal respiratory distress syndrome of course is expected to respond to surfactant therapy.

On the other hand, neonates with congenital diaphragmatic hernia seem to represent a group, in which there seems to be a poor response either to surfactant, inhaled nitric oxide and high-frequency ventilation [20]. For this specific group, a protocol for the postnatal management has been established. The data of the controlled clinical trial based on this protocol still is awaited. First analyses were presented during a national meeting (Reiss I, personal communication; GNPI Meeting, Bonn Germany; 2014) give a first impression of the complexity of this problem. There seems to be a heterogeneity even in the group of neonates with need for ECMO with respect to the distribution of single components of pathophysiology, e.g. pulmonary hypertension, pulmonary hypoplasia and myocardial failure.

Nevertheless, given these limitations, the study does provide a deep insight into the actual practices in the management of this heterogeneous group of near-term and term babies with severe respiratory failure in a pre-ECMO condition. Furthermore, the authors do address the question, if a transfer in time to ECMO center when babies $>34+0$ weeks of gestational age does offer an advantage in terms of improved survival. This critical question can only be addressed on the basis of availability of networks, offering a clear cut algorithm in the treatment of respiratory failure in this inhomogeneous group of patients as well as logistics for transfer of severely sick near-term and preterm neonates.

Data from Florida [7] indicate that the mortality can be reduced, given such a setting is established, whereas the authors of the ESPED study nicely show that a poor response to adjunctive therapy also was a strong predictor for mortality whether or not these near term preterm or term neonates received ECMO. These 
data, which were based on subjective judgments, which may limit the value of the statement, indicate highly significantly that a poor response to the adjunctive therapies should be a definite signal to discuss transfer of affected neonates to an ECMO center. It further should be used a strong argument for the centralization of neonates with prenatally diagnosed congenital diaphragmatic hernia with anticipated need for ECMO based on prenatal measurement of lung volumes, either by ultrasound or MRI techniques.

A further subgroup of patients needs to be mentioned on interpretation of the data: Intractable respiratory failure due to genetic disorders of surfactant production or metabolism [17]. Among others, inherited disorders of surfactant production (mutations of the surfactant protein B gene in most cases) as well as those regulating intra- and extracellular surfactant metabolism (ABCA3 gene and GMCSF receptor gene) have been demonstrated to be the cause for severe neonatal respiratory failure, which up to now cannot be treated successfully. Thus, before putting a neonate to ECMO, these rare causes of neonatal respiratory failure should be excluded, since successful therapy up to now has not been established [24]. The search for lifesaving new therapies either by gene manipulation or stem cells still is waiting for a solution.

A second point should be mentioned in the context preterm or term neonates with severe respiratory failure: There seems to be lack of long-term outcome studies, which are crucial to judge on the effects of new therapies or combination of established therapies for neonatal severe respiratory failure. Some centers did address this issue in neonates, e.g. with congenital diaphragmatic hernia and other well defined entities. In a review, Chiu and Hedrick [6] could demonstrate that the increase in survival in patients with congenital diaphragmatic hernia treated by ECMO or adjunctive therapies in pre-ECMO conditions is associated with an increase in long-term morbidity, especially in terms of neurologic, nutritional and musculoskeletal disorders. There is a need for carefully controlled follow-up trials in neonates treated with ECMO either for primary pulmonary or hemodynamic disorders (postsurgical cardiac ECMO). Also in this group, a substantial long term follow-up morbidity e.g. working speed, spatial ability competence and memory has been demonstrated in a Canadian study [16]. These data further underline the need for long term follow-up in near term or term neonates who were given neonatal ECMO.

\section{References}

1 Askie LM, Ballard RA, Cutter GR et al. Inhaled nitric oxide in preterm infants: an individual-patient data meta-analysis of randomized trials. Pediatrics 2011; 128: 729-739

2 Bartlett RH, Gazzaniga AB, Fong SW et al. Extracorporeal membrane oxygenator support for cardiopulmonary failure. Experience in 28 cases. The Journal of thoracic and cardiovascular surgery 1977; 73 : 375-386

3 Bennett CC, Johnson A, Field DJ et al. UK collaborative randomised trial of neonatal extracorporeal membrane oxygenation: follow-up to age 4 years. Lancet 2001; 357: 1094-1096

4 Bin-Nun A, Schreiber MD. Role of iNO in the modulation of pulmonary vascular resistance. J Perinatol 2008; 28 (Suppl 3): S84-S92
5 Carcillo JA. A synopsis of 2007 ACCM clinical practice parameters for hemodynamic support of term newborn and infant septic shock. Early Hum Dev 2014; 90 (Suppl 1): S45-S47

6 Chiu P, Hedrick HL. Postnatal management and long-term outcome for survivors with congenital diaphragmatic hernia. Prenatal diagnosis 2008; 28: 592-603

7 Clark $R H$. The epidemiology of respiratory failure in neonates born at an estimated gestational age of 34 weeks or more. J Perinatol 2005; 25: 251-257

8 Clark RH, Yoder BA, Sell MS. Prospective, randomized comparison of high-frequency oscillation and conventional ventilation in candidates for extracorporeal membrane oxygenation. J Pediatr 1994; 124: 447454

9 Cools F, Henderson-Smart DJ, Offringa $M$ et al. Elective high frequency oscillatory ventilation versus conventional ventilation for acute pulmonary dysfunction in preterm infants. Cochrane Database Syst Rev 2009, doi:10.1002/14651858.CD000104.pub3 CD000104

10 El Shahed AI, Dargaville PA, Ohlsson A et al. Surfactant for meconium aspiration syndrome in term and late preterm infants. Cochrane Database Syst Rev 2014; 12: CD002054

11 Findlay RD, Taeusch HW, Walther FJ. Surfactant replacement therapy for meconium aspiration syndrome. Pediatrics 1996; 97: 48-52

12 Finer NN, Barrington KJ. Nitric oxide for respiratory failure in infants born at or near term. Cochrane Database Syst Rev 2006, doi:10.1002/14651858.CD000399.pub2 CD000399

13 Gupta P, McDonald R, Chipman CW et al. 20-year experience of prolonged extracorporeal membrane oxygenation in critically ill children with cardiac or pulmonary failure. The Annals of thoracic surgery 2012; 93: 1584-1590

14 Henderson-Smart DJ, De Paoli AG, Clark RH et al. High frequency oscillatory ventilation versus conventional ventilation for infants with severe pulmonary dysfunction born at or near term. Cochrane Database Syst Rev 2009, doi:10.1002/14651858.CD002974.pub2 CD002974

15 Hofer N, Jank K, Resch E et al. Meconium aspiration syndrome - a 21-years' experience from a tertiary care center and analysis of risk factors for predicting disease severity. Klin Padiatr 2013; 225: 383-388

16 Ijsselstijn $H$, van Heijst AF. Long-term outcome of children treated with neonatal extracorporeal membrane oxygenation: increasing problems with increasing age. Semin Perinatol 2014; 38: 114-121

17 Nkadi PO, Merritt TA, Pillers DA. An overview of pulmonary surfactant in the neonate: genetics, metabolism, and the role of surfactant in health and disease. Molecular genetics and metabolism 2009; 97: 95-101

18 Patry C, Hien S, Demirakca $S$ et al. Adjunctive Therapies for Treatment of Severe Respiratory Failure in Newborns. Klin Padiatr 2015; 227: $28-32$

19 Pocivalnik M, Meheden SV, Griesmaier E et al. Pneumothorax during mechanical ventilation - therapeutic options in term and preterm neonates. Klin Padiatr 2013; 225: 389-393

20 Reiss I, Schaible T, van den Hout L et al. Standardized postnatal management of infants with congenital diaphragmatic hernia in Europe: the CDH EURO Consortium consensus. Neonatology 2010; 98: 354-364

21 Schaible T. Extrakorporale Membranoxygenierung (ECMO). Monatsschrift Kinderheilkunde 2011; 159: 948-954

22 Schaible T. Prognosis and therapy of congenital diaphragmatic hernia depending on the time of clinical presentation - experience of a highly specialized referral centre. Klin Padiatr 2013; 225: 218-222

23 Sweet DG, Halliday HL, Speer CP. Surfactant therapy for neonatal respiratory distress syndrome in 2013. The journal of maternal-fetal \& neonatal medicine: the official journal of the European Association of Perinatal Medicine, the Federation of Asia and Oceania Perinatal Societies, the International Society of Perinatal Obstet 2013; 26 (Suppl 2): 27-29

24 Winter J, Essmann S, Kidszun A et al. Neonatal respiratory insufficiency caused by an (homozygous) ABCA3-stop mutation: a systematic evaluation of therapeutic options. Klin Padiatr 2014; 226: 53-58 